\title{
GENERATION OF SOMATIC CELLS BY DIRECT CONVERSION - DO WE NEED PLURIPOTENT CELLS?
}

\author{
Lyle Armstrong ${ }^{1,}$, Miodrag Stojkovic ${ }^{2}$, Majlinda Lako ${ }^{1}$ \\ ${ }^{1}$ Institute of Genetic Medicine, Newcastle University, International Centre for Life, Central Parkway, \\ Newcastle upon Tyne. NE1 3BZ United Kingdom \\ ${ }^{2}$ Human Genetics, Faculty of Medicine, University of Kragujevac, Serbia
}

Received / Primljen: 06. 09. 2011.

\begin{abstract}
The pluripotency of embryonic stem cells (ESCs) makes them a potentially attractive resource for generating clinically useful somatic cells except for the problem of immune rejection. In effect, a transplant of cells differentiated from an ESC line is no different than receiving cells from the individual that would have developed from the embryo that from which the ESCs were originally derivedfrom. In view of this, it should not be surprising that the recipient's immune system could attempt to reject the incoming "foreign" cells. This is the primary reason why techniques to make individualised human pluripotent stem cells hhave been intensively investigated over the last twenty years. Initial attempts focused on the possibility of "therapeutic cloning", the deliberate creation of a human embryo by transfer of a somatic nucleus from the intended recipient into an oocyte from a human donor, with the aim of creating a tailor-made stem cell line from that embryo's inner cell mass; however, this method has not yet been successful $(1,2)$. Therapeutic cloning may indeed have been rendered obsolete by the technique of induced pluripotency $(3,4)$, and if the products of this method, namely induced pluripotent stem cells or iPSCs, are truly equivalent to ESCs, this could be the way to usher in the long promised age of personalised regenerative medicine. There are still substantial problems to overcome before this becomes reality. Even if iPSCs and ESCs are equivalent, we still need to develop reproducible methods to direct their differentiation into clinically useful cells or tissues in a cost-effective manner. Moreover, we must ensure that the resulting cells are functionally equivalent to their adult body counterparts and do not create additional health problems years after their administration. In short, there is still much work to be done.
\end{abstract}

\section{EARLY ATTEMPTS AT CELLULAR ALCHEMY}

There may be other ways to produce clinically useful differentiated cells that do not require the formation of pluripotent stem cells. This might be advantageous because pluripotency seems to compromise the cells ability to give rise to equivalent adult somatic cells. Once a genome has been reverted back to an embryonic state, it is difficult to obtain somatic cell types that differentiate from this state to function as though they were part of the adult body. Thus, how could we make the desired cell types in other ways? One possibility would be to convert one adult somatic cell type into another by reprogramming its gene expression profile (or transcriptome). Cell fusion studies suggested that the cytoplasm of ESCs was able to reprogram fibroblast nuclei into a pluripotent state (5), which supports the idea that the transcriptome of cells may be altered more readily than previously imagined. In addition, other data suggested that some somatic cells were able to change into other types under the appropriate culture conditions, which begs the question "can we convert one cell type to another simply by exposing the target cell to the cytoplasm of the other cell type?" In 2002, studies published by the laboratory of Phillipe Collas suggested that exposure of human fibroblasts and fibroblast nuclei to extracts from $T$ cells changed the transcriptome of the fibroblasts to resemble that of the T-cells (6). The technique was further developed by the transient permeabilisation of whole cells using streptolysin $\mathrm{O}$ followed by exposure to a $\mathrm{T}$-cell extract treated with RNaseI to eliminate the possibility of fibroblast transcription of transferred T-cell mRNA. Fibroblasts exposed to the extract were resealed by treatment with dilute calcium chloride and then expanded in culture. Reprogrammed cells began to express surface antigens typical of haematopoietic cells 24-60 hours after exposure to the T-cell extract, indicating that these were newly translated molecules and had not been simply carried over in the extract. Furthermore, acetylation of the promoters of the genes encoding such proteins suggested that epigenetic reprogramming of the fibroblast genome had taken place to impose the transcriptome of the T-cell, at least in part on a different genome. The reprogramming ability was not confined to T-cell extracts, as Collas' group went on to demonstrate that the treatment of primary rat fibroblasts with extracts from a rat insulinoma cell line induced expression of the pancreas-specific $P d x 1$ and insulin genes (7). This was followed by directed differentiation of pluripotent mouse ESCs into type II pneumocytes (8) and cardiomyocytes (7); however, expression of the genes upregulated by reprogramming was often tran- 
sient depending on the nature of the type of cell derived. Rat insuloma reprogrammed fibroblasts could only maintain Pdx1 and insulin gene expression for several days, although Jurkat cell extract treated 293T cells were able to maintain the new transcriptome over three months, albeit with progressive decreases in expression levels (9). This implies that the epigenetic reprogramming requirement for exogenous gene upregulation is not permanent. Conversely, there is evidence from studies from Collas' group and others that the newly imposed epigenetic modifications are heritable because activating histone modifications are detectable more than a week after extract exposure in cells that are known to divide every 24 hourshrs . Not all possible histone modifications that could constitute a stable gene expression control system were measured in these cells, and, in any case, these are more labile and subject to frequent change than the overall levels of DNA methylation at the promoters of upregulated genes. Bisulphite sequencing allows us to identify methylated cytosines in CpG dinucleotides (10), which has shown substantial demethylation of the OCT4 promoter in mouse fibroblasts treated with mouse ESC extracts. Again, the stability of this DNA methylation is uncertain and has only been analysed in detail for somatic cells reprogrammed by ESC extracts in an attempt to generate pluripotent stem cells. It is possible that reprogramming using somatic cell extracts (such as cardiomyocytes) may not lead to such extensive demethylation of target genes, which could account for the instability of the imposed transcriptome.

\section{IMPROVING DIRECT INTERCONVERSION OF SOMATIC CELLS}

The reprogramming phenomenon shown in previous studies likely resulted from the presence of cytoplasmic "factors" in the cells used to make the reprogramming extract that were not identified by Collas' or other groups. Identifying and applying these factors might generate an improved strategy for generating specific cell types as long as the factors are specific to the desired cells and capable of reprogramming. Several studies have now indicated that ectopic overexpression of isolated factors can turn one differentiated cell type into another, although this is usually within the same lineage. This means that cells derived from the mesoderm during embryogenesis tend to be able to produce only other types of mesodermal cells. The same seems to hold true for cells of the ectoderm and endoderm. Thus for the most part, these differentiations are not considered to undergo transdifferentiation.

\section{DIRECT CONVERSION TO NEURONS}

The first example of this type of conversion was the conversion of mouse and human fibroblasts into functional neurons by transfection of the former with only three neuron-specific transcription factors. Following the argument that the transcription factors that most closely defined the neuronal transcriptome, a total of 19 neuron specific genes were packaged into lentiviral vectors and used to transfect mouse tail tip fibroblasts. Those combinations of transcription factors required to induce neurogenesis were narrowed down to Ascl1, Brn2 and Myt1l (11). The fibroblasts were obtained from 3-day-old Tau-GFP and Rosa26-rtTA mice to enable the detection of neural progenitors via their expression of green fluorescent protein (GFP) under the control of the Tau promoter (a neural-specific gene). GFP expression was detectable a few days after transfection, and after FACS enrichment, these cells were able to integrate into the layers of previously cultured neonatal cortical neurons. Thus, the ability of the direct conversion cells to form functional synapses was demonstrated. Electrophysiological characteristics of the cultured Tau-GFP cells were measured by patch clamp, and they were shown to generate repetitive action potentials similar to those of mature neurons.

\section{CARDIOMYOCYTES FROM FIBROBLASTS}

The group led by Sheng Ding at the Scripps Research Institute is well known for its publications describing the influence of small molecules on the epigenetic reprogramming process of iPSC derivation, but in December 2010, they published a method for the direct conversion of mouse embryonic fibroblasts (MEFs) into differentiated cardiomyocytes. Although, in this case, they used a similar method to iPSC induction but with culture conditions designed to favour cardiogenesis. As for the neuronal conversions above, they made use of a myocardium-specific reporter gene driving LacZ expression, but transfection of the MEFs was carried out with Oct4, Sox 2 and Klf4, which would normally be expected to drive the fibroblast genome towards pluripotency when exposed to the appropriate culture conditions. The presence of cardiogenic cells was indicated by transient, widespread $\beta$-galactosidase expression, and modification of the culture medium to a progressively lower concentration of fetal bovine serum and inhibitors of JAK-STAT signalling enhanced the number of cells presenting mid-stage cardiac markers, such as Flk1, Nkx2.5 and Gata4, approximately 9 days after transfection. Late-stage cardiac markers such as cardiac troponin appeared after 11 days, and areas of spontaneously contracting tissue appeared on day 15 . Addition of the cardio-inductive growth factor Bone Morphogenetic Protein 4 (BMP4) increased the number of contracting areas by nearly 150 -fold (12). The contraction rate was variable, ranging from 4-130 beats per minute, but, they also exhibited calcium transient and electrophysiological action potentials similar to those reported by other groups, which suggested a primarily atrial phenotype for the cardiomyocytes generated in this work.

The method used to generate cardiomyocytes in this manner is interesting because it suggests that the fibroblasts may have been reprogrammed to a pluripotent state 
before re-differentiating down a cardiogenic pathway. Although this cannot be formally ruled out, it is an unlikely scenario because commitment to the cardiac lineage occurs soon after transfection with the lentiviral vectors, and typical indicators of the onset of a pluripotent state, such as the upregulation of the endogenous copy of Nanog, are scarcely observable. Examination of the latter point using fibroblasts obtained from mice carrying a Nanog-GFP reporter construct suggested that pluripotent cells were not present at the time points from which cardiac markers appeared in the cell cultures. Bonafide markers of pluripotency only appeared under growth conditions that promoted formation of iPSCs and at much later time points after transfection. Taken together these observations point to the emergence of cardiac lineage cells from nonpluripotent precursors.

\section{MAKING BLOOD}

A key observation made by the Bhatia group was the expression of the pan-haematopoietic marker CD45 in some colonies of cells during iPSC derivation. Approximately ten days after transfection with OCT4, SOX2, KLF4 and / or $c-M Y C$, the cells no longer resembled the parent fibroblasts but did not have any similarity to genuine iP$\mathrm{SCs}$. The presumption is that these are cells at some intermediate stage of dedifferentiation, but it is not yet clear if reprogramming entails progressive reversal of lineage commitment or erasure of the epigenetic information controlling cell identity over a short timeframe. Bhatia's suggestion is that the bulk of the epigenetic erasure is performed by the ectopic expression of OCT4, but this factor cannot induce pluripotency on its own and needs SOX2 and KLF4, at least, to complete the process. No markers of pluripotency such as Tra-1-60, which can normally be detected by day 21 of normal iPSC induction, were observed, and OCT4 transduced fibroblasts were unable to generate teratomas in immunodeficient mice (13). OCT4 overexpression produces detectable numbers of CD45 expressing cells 21 days after transfection, and although this method is a departure from the transcription factor screening technique employed to select neurogenic factors, it presents a tantalising possibility of making blood cells for autologous transplant into adults.

Gene expression analysis of the OCT4-CD45-expressing cells (enriched by FACS for the CD45 cell surface antigen) showed a high degree of similarity to haematopoietic progenitors derived from peripheral blood or umbilical cord blood, which implies a possible haematopoietic function for these cells. Expansion of OCT4-CD45 cells in the presence of cytokines that support haematopoietic progenitor development gave rise to progeny expressing surface antigens characteristics of a range of differentiated haematopoietic cell types such as monocytes (CD14) and myeloid progenitors (CD33 and CD13) in addition to cells with typical neutrophil, eo- sinophil and basophil morphologies. OCT4-CD45 cells transplanted into myeloablated immunodeficient NOD/ SCID IL2Ryc-null mice contributed largely to the development of cells with a myeloid phenotype, although the level of engraftment was comparable to that obtained with umbilical cord blood progenitors. Importantly, the primary engrafted OCT4-CD45 cells showed only limited ability to engraft a secondary NOD/SCID IL2Ryc-null mouse suggesting that their ability to undergo indefinite expansion is reduced compared with haematopoietic progenitors derived from pluripotent cells. This implies that the OCT4-CD45 cells may be a safer alternative in view of the tendency of pluripotent derived haematopoietic cells to undergo leukemic transformation. Erythropoiesis from the OCT4-CD45 cells provided an interesting contrast to pluripotent stem cells in that treatment with erythropoietin (a growth factor that induces- erythropoiesis in vitro and in vivo) resulted in the production of CD71 expressing erythroblasts expressing glycophorin A and the adult $\beta$-globin protein. Despite this, the OCT4CD45 cells were only able to complete the differentiation of the megakaryocyte-platelet lineage, and erythrocytes were not detected. Moreover, lymphopoiesis was not observed despite expression of CD34 in 25\% of the OCT4-CD45 cells, which indicated the possible presence of more primitive haematopoietic progenitor cells that were capable of multilineage differentiation. Apart from the expression of CD45 following OCT4 transfection, the development of the subsequent haematopoietic cell types seemed to depend on the presence of haematopoiesis promoting cytokines. Therefore, it is possible that the cell types detected in this study arose from a haematopoietic progenitor or more committed blood cell type present in the dermal fibroblast samples.

\section{HEPATOCYTES}

In a similar approach to the screen for inducers of neurogenesis, combinations of the transcription factors $H n f 4 \alpha$, Foxa1, Foxa 2 and Foxa 3 were shown to convert mouse embryonic stem cells and adult fibroblasts into hepatocytes. Two weeks after transfection with individual pools of two factors, the cells were replated onto collagen. Three weeks after the replating step, clusters of cells with an epithelial morphology and normal karyotype appeared and could be maintained in culture for several passages (referred to as iHep cells) (14). These did not express markers typical of fibroblasts, but they were strongly positive for E-cadherin, albumin and the canalicular membrane protein multidrug resistance protein, Mrp2,; all of which are typical of hepatocytes. iHep cells had a similar transcriptomic profile to ex vivo-derived hepatocytes and showed several functional similarities such as production of urea, synthesis of triglycerides and cytochrome P450 activity. In contrast to the haematopoietic data generated by Bhatia's group, iHep cells had a greater similarity to terminally differentiated hepato- 
cytes because there was little evidence of more primitive endodermal progenitors (such as pancreatic or intestinal cells) after transfection with any of the transcription factor combinations. There was also little convincing evidence of bi-potent hepatic progenitor cells (cholangiocytes) due to lack of cytokeratin 7 expression. Regardless, iHep cells appeared to function in vivo. Hepatocytes isolated from the adult mouse liver are able to reconstitute the hepatic tissues of mice deficient for the enzyme fumaryl acetoacetate hydrolase (FAH), which are used to model liver injury (15). One month after intrasplenic injection of iHep cells into FAH-/- mice, FAH-expressing hepatocytes had engrafted and reconstituted the hepatic tissues resulting in rescue from liver failure. Conversely, all the FAH-/- mice transplanted with the untransfected fibroblasts (i.e., non-iHep cells) died within 27 days.

As with the other examples of direct conversion described in this review, there is still the possibility that iHep cells could have been derived from endodermal progenitor cells present in the fibroblast population used for transfection. This was particularly true for the earliest experiments of this research group, as they made use of MEFs obtained by dissection of E12.5 mouse embryos. Because it is not easy to completely remove the embryonic liver and other components of the digestive system from such embryos, it is thus possible that endodermal progenitor cells were present in the MEF culture; however, iHep cells were also obtained from adult mouse dermal fibroblasts. As mesenchymal stem cells (MSCs) have been shown to transdifferentiate into hepatocyte-like cells (16), another consideration is the possible presence of MSCs in the fibroblast cultures. However, against this possibility are the observations that iHep cells arise with similar frequency from both enriched MSCs and fibroblast cultures.

\section{MECHANISMS OF DIRECT CONVERSION FROM FIBROBLASTS}

From the limited examples of direct conversion in the scientific literature, it is difficult to hypothesise about possible mechanisms that may reprogram one cell type into another. Two of the above examples involve transfection of at least one transcription factor associated with the pluripotent state, and although the authors of these publications have gone to considerable lengths to rule out the reprogramming of the fibroblast genome back to a pluripotent state followed by re-differentiation to the target cell type, it is still possible that pluripotency transcription factor may contribute to direct conversion. The capacity of Oct4 for epigenetic reprogramming has been well documented, and iPSCs have been generated from neural stem cells (NSCs) from the adult subventricular zone by transfection of Oct4 alone (17, 18 ), implying that the Oct4 gene product is able to select some (or perhaps all) of its binding sites within the
NSC genome. In addition, iPSCs can be generated by a combination of Oct4 transfection and treatment with small molecules that inhibit certain chromatin-modifying enzymes (19). In view of these data, Oct4 could be a master regulatory gene of the pluripotent phenotype, but it requires other gene products to induce true pluripotency. SinceAs zygotic deletion of Oct4 causes mouse epiblast cells to undergo extensive chromatin compaction, it is possible that one of the functions of Oct4 is maintenance of an "open" chromatin conformation (20). This implies (and other groups have speculated) that Oct4 may perform this task by ensuring that the epigenetic modifications associated with chromatin compaction are not imposed upon the pluripotent genome. The converse of this argument may also be that it ensures the removal of such modifications from a somatic genome and imposes a much greater degree of differentiation plasticity upon the cell. This is not the same pluripotency as we find in ESCs, but it may allow more access to transcription factors that favour differentiation down several lineages and expression of a wider range of transcription factors associated with varied cell identities. The conditions under which we grow Oct4-expressing cells may then favour the survival of some somatic cell types over others, or they may induce signal transduction mechanisms that instruct lineage-specific differentiation. Both of these possibilities have been invoked to explain the direct conversion of fibroblasts to cardiomyocytes and haematopoietic cells, indicated in the publications described earlier.

It is more difficult to explain why ectopic expression of Hnf4 $\alpha$ with one of more of the Foxa genes is sufficient to convert fibroblasts into iHep cells; however, potential clues may lie in a possible chromatin-modifying function of the Foxa gene family. Mammals have three seemingly unlinked FoxA genes (FoxA1, FoxA2, and FoxA3), of which FoxA2 has functions closely linked to endoderm development $(21,22)$. Ectopic expression of FoxA2 promotes endoderm development in ESCs (23), and it has also been shown that FoxA genes, as part of the more general class of Fox or forkhead box genes, function in diverse developmental and signal transduction mechanisms (24). A major aspect of this function seems to be maintenance of an open chromatin conformation that is reminiscent of the similar, albeit probably more extensive, function of Oct4. The purpose of this appears to be pre-conditioning of the genomes of endoderm progenitor cells to activate liver, pancreas or other tissue-specific genes, and for this reason, FoxA genes have been termed "pioneer" factors. In view of this, it is possible that ectopic expression of one or more of the FoxA genes needed to induce iHep cell formation may be the determinant of epigenetic reprogramming of the fibroblast genome.

Another possibility is that fibroblasts have an inherent plasticity that allows them to differentiate more readily. Although not extensive, there is evidence in the literature suggesting that human dermal fibroblasts can 
differentiate into cells representative of all three embryonic germ layers when cultured under appropriate conditions (25). The cell types reported in this recent publication include neuronal (indicated by nestin and $\beta$-III-tubulin expression), immature myoblast-like cells (Myf5 and Desmin expression) and possible insulin-synthesising cells that are indicative of endoderm. There are additional data indicating that fibroblasts are capable of multi-lineage differentiation, and we cannot formally rule out the presence of progenitor or tissue-specific stem cells in the fibroblast culture. However, this apparent plasticity is worth bearing in mind for future studies of direct conversion.

\section{CONCLUDING REMARIKS}

Induced pluripotent stem cells are relatively easy to generate and expand in culture, but we have to be certain they can produce safe, clinically useful and economically viable cell products before they can have a major impact on regenerative medicine. Therefore, it is possible that direct conversion methods may have advantages in terms of the possibilities of generating cells that have greater similarity to those found in the adult body. There are enormous hurdles to overcome: the phenomenon is restricted to a small set of very recent observation, and there are no guarantees that this concept will be universally applicable to all clinically desirable cell types. Furthermore, we have no data concerning the mutational load present in directly converted cells. If these are derived from tissues of older individuals, they could have accumulated significant levels of damaged DNA in both the nuclear and mitochondrial genomes. They may have shorter telomeres, and as some publications suggest, epigenetic changes may accumulate as a function of organismal age $(26-29)$. Although iPSCs may be able to reset damage such as telomere lengths, we cannot be certain that direct conversion will do this .

\section{REFERENCES}

1. Stojkovic M, Stojkovic P, Leary C, Hall VJ, Armstrong L, Herbert M, Nesbitt M, Lako M, Murdoch A. (2005) Derivation of a human blastocyst after heterologous nuclear transfer to donated oocytes. Reprod Biomed Online. 11(2):226-31.

2. Hwang WS, Ryu YJ, Park JH, Park ES, Lee EG, Koo JM, Jeon HY, Lee BC, Kang SK, Kim SJ, Ahn C, Hwang JH, Park KY, Cibelli JB, Moon SY.(2004) Evidence of a pluripotent human embryonic stem cell line derived from a cloned blastocyst. Science. 303(5664):1669-74 (retracted in Kennedy D. Science. 311(5759):335)

3. Yu J, Vodyanik MA, Smuga-Otto K, AntosiewiczBourget J, Frane JL, Tian S, Nie J, Jonsdottir GA, Ruotti V, Stewart R, Slukvin II, Thomson JA (2007) Induced pluripotent stem cell lines derived from human somatic cells. Science. $\mathbf{3 1 8 ( 5 8 5 8 ) : 1 9 1 7 - 2 0}$

4. Takahashi K, Yamanaka S (2006) Induction of pluripotent stem cells from mouse embryonic and adult fibroblast cultures by defined factors. Cell. 126(4):663-76

5. Tada, M., Takahama, Y., Abe, K., Nakatsuji, N. \& Tada, T.(2001) Nuclear reprogramming of somatic cells by in vitro hybridization with ES cells. Curr. Biol. 11: 1553-1558

6. Håkelien AM, Landsverk HB, Robl JM, Skålhegg BS, Collas P.(2002) Reprogramming fibroblasts to express T-cell functions using cell extracts. Nat Biotechnol. 20(5):460-6

7. Gaustad KG, Boquest AC, Anderson BE, Gerdes AM, Collas P.(2004) Differentiation of human adipose tissue stem cells using extracts of rat cardiomyocytes. Biochem Biophys Res Commun. 314(2):420-7.

8. Qin M, Tai G, Collas P, Polak JM, Bishop AE.(2005) Cell extract-derived differentiation of embryonic stem cells. Stem Cells. 23(6):712-8

9. Håkelien AM, Gaustad KG, Taranger CK, Skålhegg BS, Küntziger T, Collas P.(2005) Long-term in vitro, celltype-specific genome-wide reprogramming of gene expression. Exp Cell Res. 309(1):32-47.

10. Clark SJ, Harrison J, Paul CL, Frommer M.(1994) High sensitivity mapping of methylated cytosines. Nucleic Acids Res. 22(15):2990-7

11. Vierbuchen T, Ostermeier A, Pang ZP, Kokubu Y, Südhof TC, Wernig M (2010) Direct conversion of fibroblasts to functional neurons by defined factors. Nature.463(7284):1035-41

12. Efe JA, Hilcove S, Kim J, Zhou H, Ouyang K, Wang G, Chen J, Ding S. (2011) Conversion of mouse fibroblasts into cardiomyocytes using a direct reprogramming strategy. Nat Cell Biol. 13(3):215-22

13. Szabo E, Rampalli S, Risueño RM, Schnerch A, Mitchell R, Fiebig-Comyn A, Levadoux-Martin M, Bhatia M.(2010) Direct conversion of human fibroblasts to multilineage blood progenitors. Nature. 468(7323):521-6

14. Sekiya S, Suzuki A.(2011) Direct conversion of mouse fibroblasts to hepatocyte-like cells by defined factors. Nature. 475(7356):390-3

15. Li F, Liu P, Liu C, Xiang D, Deng L, Li W, Wangensteen K, Song J, Ma Y, Hui L, Wei L, Li L, Ding X, Hu Y, He Z, Wang X. (2010) Hepatoblast-like progenitor cells derived from embryonic stem cells can repopulate livers of mice. Gastroenterology. 139(6):2158-2169

16. Snykers S, De Kock J, Tamara V, Rogiers V. (2011) Hepatic differentiation of mesenchymal stem cells: in vitro strategies. Methods Mol Biol. 698:305-14.

17. Deleidi M, Cooper O, Hargus G, Levy A, Isacson O.(2011) Oct4-induced reprogramming is required for adult brain neural stem cell differentiation into midbrain dopaminergic neurons. PLoS One. 6(5):e19926.

18. Kim JB, Zaehres H, Araúzo-Bravo MJ, Schöler HR.(2009) Generation of induced pluripotent stem cells from neural stem cells. Nat Protoc. 4(10):1464-70 
19. Li Y, Zhang Q, Yin X, Yang W, Du Y, Hou P, Ge J, Liu C, Zhang W, Zhang X, Wu Y, Li H, Liu K, Wu C, Song Z, Zhao Y, Shi Y, Deng H. (2011) Generation of iPSCs from mouse fibroblasts with a single gene, Oct4, and small molecules. Cell Res. 21(1):196-204

20. Ahmed K, Dehghani H, Rugg-Gunn P, Fussner E, Rossant J, Bazett-Jones DP(2010) Global chromatin architecture reflects pluripotency and lineage commitment in the early mouse embryo. PLoS One. 5(5):e10531

21. Zaret K.(1999) Developmental competence of the gut endoderm: genetic potentiation by GATA and HNF3/ fork head proteins. Dev Biol. 209(1):1-10

22. Davidson EH, Erwin DH.(2006) Gene regulatory networks and the evolution of animal body plans. Science. 311(5762):796-800

23. Ishizaka S, Shiroi A, Kanda S, Yoshikawa M, Tsujinoue H, Kuriyama S, Hasuma T, Nakatani K, Takahashi K.(2002) Development of hepatocytes from ES cells after transfection with the HNF-3beta gene. FASEB J. 16(11):1444-6

24. Katoh M, Katoh M.(2004) Human FOX gene family (Review). Int J Oncol. 25(5):1495-500.

25. Osonoi M, Iwanuma O, Kikuchi A, Abe S.(2011) Fibroblasts have plasticity and potential utility for cell therapy. Hum Cell. 24(1):30-4

26. Li Z, Liu C, Xie Z, Song P, Zhao RC, Guo L, Liu Z, Wu Y. (2011) Epigenetic dysregulation in mesenchymal stem cell aging and spontaneous differentiation. PLoS One. 6(6):e20526

27. Klauke K, de Haan G (2011) Polycomb group proteins in hematopoietic stem cell aging and malignancies. Int J Hematol. 94(1):11-23.

28. Pollina EA, Brunet A.(2011) Epigenetic regulation of aging stem cells. Oncogene. 30(28):3105-26

29. Chambers SM, Shaw CA, Gatza C, Fisk CJ, Donehower LA, Goodell MA.(2007) Aging hematopoietic stem cells decline in function and exhibit epigenetic dysregulation. PLoS Biol. 5(8):e201. 